RESPONSE

\title{
Evidence for light-entrainment-induced switching between depression- \& mania-relevant behaviors in mice
}

\author{
Neuropsychopharmacology (2019) 44:1679-1680; https://doi.org/10.1038/s41386-019-0338-3
}

We appreciate Rosenthal and McCarty's careful read of our study [1], demonstrating that mice kept in short- and long-active (SA and LA respectively) photoperiods (19Light;5Dark, 5Light:19Dark respectively), exhibit neurotransmitter and behavioral switching. The mice with reduced dopamine transporters (DAT) exhibit a hypersensitivity to these photoperiods, recreates common observations in bipolar disorder (BD), AKA seasonality, with mania observed in patients as they enter LA (summer), and depression in patients as they enter SA (winter) photoperiods. Given that unmedicated BD patients exhibit lower expression of DAT, this work highlights seasonality as perhaps the origin of/contributor to switching between states in BD. The authors' commentary raises 3 concerns regarding such an interpretation. We appreciate the opportunity and space here to provide clarity.

Concern 1: C57BL/6J mice do not possess melatonin, hence why would they exhibit different behaviors with shifted photoperiods? C57BL/6 mice clearly exhibit shifts in behavior with changing photoperiods, both in our and others' studies [2]. This premise is supported by findings that "melatonin is not required to initiate or even maintain circadian rhythms" [3]. The superchiasmatic nucleus $(\mathrm{SCN})$ is the brain region where light information is first processed. The SCN stores photoperiodic information by producing an electrical activity waveform, triggering behavioral activity (offset at $>50 \%$, onset at $<50 \%$; [4]. Peak activity of the SCN's electrical activity pattern is compressed in a SA photoperiod, but dilated for LA photoperiods [5]. We observed neurotransmitter switching in the paraventricular nuclei (PVN), with somatostatin (SST) expressed during SA and tyrosine hydroxylase (TH) expressed during LA photoperiods consistent with rats [6]. The PVN is the next structure relaying light information and the SCN-PVN axis is essential for relaying photoperiodic information to other parts of the body, with PVN volume fluctuating seasonally [7], unlike other brain regions. Further, removing the pineal gland - source of melatonin - exerts only limited effects on mammalian circadian rhythmicity [8]. Hence, the concern that lack of melatonin may invalidate our interpretations is inconsistent with current understanding of photoperiodic entrainment. We suggest the mechanism underlying changes in our observations are not melatonin-dependent. Interestingly, and in support of our overall interpretation for BD, a meta-analysis of melatonin studies reveal no utility as a treatment for BD [9], despite light exposure being useful [10].

Concern 2: The change in photoperiod length was insufficient for mice to entrain behaviorally and neurophysiologically, citing a 'need' for 30 days for 'consistent' waveform changes in the circadian rhythm. Our study did not investigate consistency, but the transition to altered photoperiods. It is the transition to winter or summer that seems to affect people with BD more, rather than their prolonged presence, a separation we intend to explore further. The fact that the same behaviors and neurotransmitter switching seen in our publication (immobility/open arm entries and TH/SST balance) replicated 7 days of entrainment in rats [6] roughly the timeline needed for human photoperiodic entrainment - strongly supports this cross-species phenomenon.

Concern 3: use of nomenclature. We utilized SA and LA because it represents the activity time of the animal, since the mice are nocturnal it appeared the simplest way to convey consistency with other diurnal organisms, especially with changes in activity as occurs in seasonal photoperiods in humans even with electric lighting. Given the primary concern of the work was an attempt to explain observations in human BD phenomenon, we felt it best to create cross-species consistent nomenclature, as our laboratory and others continually strive toward (e.g., testing nocturnal animals only in their dark (active) photoperiod vs. light period just because humans are tested in the light). Certainly, it offers an opportunity for consistency across species as opposed to the confusion of delineating nocturnal/diurnal comparisons. We appreciate the concern raised, however, as it provides further opportunity to explain our reasoning.

In summary, we appreciate the attention the authors brought to our study, the concerns raised provided greater opportunity to delineate our approach. We hope this response goes a way toward clarifying that approach.

\section{ADDITIONAL INFORMATION}

Competing interests: The authors declare no competing interests.

Publisher's note: Springer Nature remains neutral with regard to jurisdictional claims in published maps and institutional affiliations.

Jared W. Young ${ }^{1,2}$, Zackary A. Cope ${ }^{1}$, Benedetto Romoli ${ }^{1}$, Esther Schrurs ${ }^{1,3}$, Aniek Joosen ${ }^{1,3}$, Jordy van Enkhuizen ${ }^{1}$, Richard F. Sharp ${ }^{1}$ and Davide Dulcis ${ }^{1}$

${ }^{1}$ Department of Psychiatry, University of California San Diego, 9500 Gilman Drive MC 0804, La Jolla, CA 92093-0804, USA; ${ }^{2}$ Research Service, VA San Diego Healthcare System, San Diego, CA, USA and

${ }^{3}$ Division of Pharmacology, Utrecht Institute for Pharmaceutical Sciences, Utrecht University, Utrecht, The Netherlands Correspondence: Jared W. Young (jaredyoung@ucsd.edu) or Davide Dulcis (ddulcis@ucsd.edu)

\section{REFERENCES}

1. Young JW, Cope ZA, Romoli B, Schrurs E, Aniek J, van Enkhuizen J, et al. Mice with reduced DAT levels recreate seasonal-induced switching between states in bipolar disorder. Neuropsychopharmacology. 2018;43:1721-31.

2. Casiraghi LP, Oda GA, Chiesa JJ, Friesen WO, Golombek DA. Forced desynchronization of activity rhythms in a model of chronic jet lag in mice. J Biol Rhythms. 2012;27:59-69.

3. Gandhi AV, Mosser EA, Oikonomou G, Prober DA. Melatonin is required for the circadian regulation of sleep. Neuron. 2015;85:1193-9.

These authors contributed equally: Jared W. Young, Davide Dulcis

Received: 29 January 2019 Revised: 30 January 2019 Accepted: 1 February 2019

Published online: 20 February 2019 
Evidence for light-entrainment-induced switching between depression. . .

$\mathrm{J} W$ Young et al.

1680

4. Houben T, Deboer T, van Oosterhout F, Meijer JH. Correlation with behavioral activity and rest implies circadian regulation by SCN neuronal activity levels. J Biol Rhythms. 2009;24:477-87.

5. Coomans CP, Ramkisoensing A, Meijer JH. The suprachiasmatic nuclei as a seasonal clock. Front Neuroendocrinol. 2015;37:29-42.

6. Dulcis $D$, Jamshidi $P$, Leutgeb $S$, Spitzer NC. Neurotransmitter switching in the adult brain regulates behavior. Science. 2013;340:449-53.

7. Hofman MA, Swaab DF. Seasonal changes in the suprachiasmatic nucleus of man. Neurosci Lett. 1992;139:257-60.
8. Morin LP. Age, but not pineal status, modulates circadian periodicity of golden hamsters. J Biol Rhythms. 1993;8:189-97.

9. De Crescenzo F, Lennox A, Gibson JC, Cordey JH, Stockton S, Cowen PJ, et al. Melatonin as a treatment for mood disorders: a systematic review. Acta Psychiatr Scand. 2017;136:549-58.

10. Geoffroy PA, Abbassi E, Maruani J, Etain B, Lejoyeux M, Amad A, et al. Bright light therapy in the morning or at mid-day in the treatment of non-seasonal bipolar depressive episodes (LuBi): study protocol for a dose research phase I / II trial. Psychiatry Investig. 2018;15:1188-202. 